\title{
AR \& VR Marketing: when and where?
}

\author{
Cătălin Ionuț SIL VESTRU \\ Bucharest University of Economic Studies, Bucharest, Romania \\ catalin@ase.ro \\ Ana Maria IFRIM \\ Titu Maiorescu University, Bucharest, Romania \\ amifrim@gmail.com \\ Ionica ONCIOIU \\ Titu Maiorescu University, Bucharest, Romania \\ nelly_oncioiu@yahoo.com \\ Marian-Ernuț LUPESCU \\ Bucharest University of Economic Studies, Bucharest, Romania \\ marian.lupescu@csie.ase.ro \\ Steliana RAMIDO \\ Bucharest University of Economic Studies, Bucharest, Romania \\ steliana.rodino@yahoo.com
}

\begin{abstract}
Marketers have been trying to find the means to innovate and introduce technology as much as they can in their line of work in order to convince consumers to acquire their products or services and due to recent outcomes, it has become harder for certain fields to benefit from conventional marketing strategies and implementations. Augmented Reality and Virtual Reality have the possibility to help fields that are overlooked in Online based marketing and even bring new light to presenting products and services to consumers. In this article, we will try to identify the advantages and disadvantages of using these technologies in marketing strategies, where they have been used successfully and where they can be used to innovate. We will be reviewing research papers that have been published in the field of virtual reality and augmented reality that are connected with marketing strategies with an emphasis on tourism, education and marketing, presenting the findings and relating our opinions on further developing these aspects.
\end{abstract}

Keywords: Augmented Reality, Virtual Reality, Marketing innovation, Education, COVID -19, Tourism, Digital Technologies.

\section{Introduction}

In the last year, due to the COVID-19 pandemic, there has been an increasing number of issues related to workforce displacement, unemployment, businesses going bankrupt (EuroStat, 2021) and other such unfortunate events. Some fields of work have been affected more than others, such as agriculture and tourism, mainly due to the restrictions that have been implemented. Other businesses managed to avoid such outcomes through the implementation of work-from-home implementations, where the employer and employee had work relations through the use of technology via a plethora live communication software tools and hardware resources.

Thus, many people have started to use technology more and more in order to fulfill their work obligations, their supply need, some of their entertainment needs, some through online 
streaming services and others through mobile applications. The question arises in regards to the possibility of utilizing technology in order to help fields that require the presence of workers in order to operate, such as tourism or agriculture.

There have been studies in regards to using Augmented Reality and Virtual Reality in various fields that include medical (Silva, Southworth, Raptis, \& Silva, 2018; Joda, Gallucci, Wismeijer, \& Zitzmann, 2019), education (Cuendet, Bonnard, Do-Lenh, \& Dillenbourg, 2013), serious gaming and many others. In addition to the educational purposes of using Virtual Reality and/or Augmented Reality, there have also been studies in the field of marketing (Rauschnabel, Felix, \& Hinsch, Augmented reality marketing: How mobile AR-apps can improve brands through inspiration, 2019) where either mobile applications (Sung, 2021) are used or virtual reality for promoting various elements.

As such, we would like to identify the positives and negatives of these technologies and then explore the possibility of implementation of fields that suffer from lack of personnel and lack of promoting in these times.

\section{Augmented reality}

Augmented reality, or AR, refers to the use of technologies in order to project materials of digital nature onto real world objects (Cuendet, Bonnard, Do-Lenh, \& Dillenbourg, 2013; Persefoni \& Tsinakos, 2016). Although this definition is a summary of a technology that encompasses a large spectrum of other technologies, that have been initially summarized by Paul Milgram and Fumio Kishino (Milgram \& Kishino, 1994). This technology has seen more and more implementation via mobile devices, although there have been various implementations throughout the years either for educational purposes that have developed TUI's (tangible user interfaces) or entertainment.

Mainstream media has first had contact with Augmented Reality in 2013 through the presentation made by Google, in which they presented a pair of glasses that had rudimentary elements that were projected on a small screen in the view of the user. After this, Microsoft also presented their approach on this technology through their HoloLens products in 2016, furthering the public knowledge of this technology. Recently, mobile phone devices from various brands utilize this technology for socializing purposes, such as Samsung AR Zone or Apple AR. The implementation for mobile devices implies the use of the device's camera and display to overlay various digital elements over the elements that the camera perceives and mix them on the user's screen.

A mainstream application that made use of this technology for marketing purposes to great effect was Pokemon Go, which is developed by Niantic in 2016 (Rauschnabel, Rossmann, \& tom Dieck, 2017). These elements combined and many others have made it possible for AR to be publicly visible and used more frequently by day to day people, outside the boundaries of research teams or closed doors. As such, applications have been made for consumers based on this technology that relate to virtual mirrors, virtual home decoration, and virtual make-up and so on.

The use of this technology in marketing will become more and more of a necessity due to the rising numbers of people that are using mobile devices and how fast technology advances. As mentioned before we will start by discussing the main advantages and disadvantages of using this technology, starting out with the advantages:

- Availability - Since the invention of the smartphone, in the Apple implementation, presented by Steve Jobs, mobile devices have been in a constant evolution. Starting from screen real-estate and ending with component performance, these devices have been replacing conventional

means of browsing the internet or accessing information, and have even shaped the marketing 
paradigm of web-design, since 2018, when mobile-first approaches were generally recommended when it came to device compatibility of a website. And taking into account that AR can be implemented in with technology that requires a camera and a display in order to mix both elements, the real and the digital, to obtain an augmented reality, the wide availability of mobile phones makes it an accessible technology for the user;

- Cost effectiveness - Since the users perceives the virtual world that is shown to him through the lens of his or her phone or mobile device, marketing developers and experts are not bound to the need of having to produce physical products or to come up with creative means to achieve results on a strict budget. Costs are reflected more in staff and technology use for development. Assets can be reused or reskinned for other purposes and the general cost of an application usually is lower than conventional marketing tools;

- Reach - As mentioned before, in the availability section, technology has advanced in such a way that at the time of writing this article, according to statistics there are an approximate of 14.02 billion mobile devices worldwide (Statista, 2020). These numbers will be in continuous growth, and as such it is safe to estimate that more than $70 \%$ of the world population owns a mobile device. With this in mind, the reach of a marketing campaign made through a mobile application that has certain users targeted is higher than that of conventional promotional strategies;

These are the main advantages of using AR in marketing that we have identified, although there can be aspects to discuss in regards creativity and sustainability, but those are generally available when discussing virtual technologies. There are also some disadvantages in using this technology, and we would like to mention the following as being the most pressing ones:

- Digital skill gap - when thinking or discussing the use of technology, the tendency is to mention the fact that it is widely available and easy to use, but studies show that more than $40 \%$ of Europeans lack basic digital skills (European Commission, 2017). This translates into the fact that there is an age-based skill divide when it comes to digital skills and literacy. Having less people that are capable of using technology in its basic forms has a negative impact on whether a certain technology will or will not be able to reach that target group;

- Security and privacy - as with all technologies, the risk of malicious intent from third parties is always present, whether it comes in the form of malicious software that is installed through the main application, or hijacking the cameras of the user and affecting their privacy etc. There are also concerns from users that are sceptic in regards to how their information is being used, and if it is not clearly stated by the application developer, users will perceive it as a privacy liability. Although this last part is directly linked with digital literacy, meaning that the less tech savvy a person is, the lower the probability that they will raise digital privacy issues.

These are the main disadvantages that have been identified with the use of this technology in marketing.

Having explored the positive and negative aspects of the technology, we would like to propose using this technology through a real world implementation of augmented posters, fliers and panels. This implementation is based of technology known as augmented photography (Zöllner, Becker, \& Keil, 2010) or augmented images, which can take advantage of Google's AR Core SDK or other such technologies. This implementation will be mainly based on mobile applications that already use an AR engine, for example Pokemon GO or Minecraft Earth, although we would rather have specific applications designed for different purposes, but taking into account that AR is mostly used in mobile gaming compared to other fields of mobile use, we will use these two as the main examples. The implementation will focus around having conventional marketing 
materials that are modified in order to be visible in different ways through the use of a device. Having a plain aspect when viewed without a mobile phone / application and then when the user has an AR application opened, that same marketing material will be augmented and thus captivate the attention of the user. This usage is possible with current day technology, though it is restricted to having an AR interpretation application developed.

The possibilities of implementation of such technologies are various, depending on the target group of the marketing strategy, and it can range from basic applications to ones that include audio and haptic feedback. The method of implementing AR marketing (Sung, 2021) has great impact on customer response and on the effectiveness of the campaign. As the authors mention in their work, there is a direct link between the quality of the AR advertisement and the overall user experience and behavioral intentions. The higher the quality and satisfaction in regards to the AR, the greater the impact it has on the consumer.

Of course, there are limitations when it comes to the use of this technology, due to the inert need to augment objects around, or make use of them in order to transmit information towards the consumer. Various fields have better success rates than others, such being the case with tourism before and after the legal aspects in regards to general lockdowns of countries during a pandemic situation.

Augmented Reality had implementation variations through the use of applications that were used to deliver consumers towards certain points of interest during their visit in specific locations. These applications work through a task system that rewards the user for completing them. Through such an application, the user generally uses the application to identify certain items around them in a location that is specified by the application, photograph them and earn points or complete the task in order to advance. Such treasure hunt based applications are appealing towards younger audiences and have had success, but in the current context such an implementation of AR is not viable due to traveling constraints. This is were Virtual Reality can be implemented in order to improve or rather, provide an experience for users.

\section{Virtual reality}

Virtual reality, or VR, can be defined as the use of computer technology to create an interactive three-dimensional world in which the objects have a sense of spatial presence (Kardong-Edgren, Farra, Alinier, \& Young, 2019). There are also other definitions for this technology, which the previous authors state in their work, but we consider that this definition is suitable for our research. The use of virtual reality is present in most medical based education programs in order to provide medical students with the right assets to train in order to gain better knowledge of the human body and gain competences in fields that normally would require resources that are difficult to provide.

In early 2020, the world has been affected by the COVID-19 pandemic that had gradually all countries close borders, restrict human interaction, and travel to a minimum. This has had a grave impact on tourism in regards to income generation, with estimates that countries like Italy losing 7.7 Billion Euros by the end of last year, decreases in occupancy in Paris by a percentage of more than $80 \%$ and an estimate between $\$ 63$ and $\$ 113$ Billion being lost on passenger transport in the last year (Andreea et al., 2021). Although, in regards to the impact that the lack of mobility in tourism has had, some aspects were positive such as lower congestion, lower rate of destruction of cultural icons and ecosystem degradations etc. Thus, we would like to mention the possibility of using Virtual Reality in order to promote touristic attractions and implement marketing solutions inside this virtual world. 
As mentioned in the definition, the world has a sense of spatial presence, which allows developers to manipulate what the users see in the world. With such freedom in regards to creation, there have been use cases for VR in tourism where, due to the degradation of the touristic attraction, heritage sites have been 3D scanned and rebuilt in VR (Toubekis, et al., 2017). This is just one use case of preserving and using Virtual Reality in order to promote the virtual visiting of an attraction. There are also attractions that have been rebuilt in virtual reality due to disasters that have happened, with the use of laser tracing and virtual worlds that were built around the existing attraction, such as Notre Dame de Paris. As seen, the idea of virtual tours is not new, and has been tried and tested throughout the world, be it due to the necessity of preservation or that of reconstruction.

Virtual worlds can be visited, depending on the complexity of the software solution, with the use of various equipment, ranging between a VR headset that can use a mobile device as a screen to project the image through its lenses in order to allow the user to see the 3D world to fullfledged VR solutions that include remote controllers and sensors such as OCULUS Rift/Quest, HTC Vive or PlayStation VR to name a few. The user experience varies based on the technology that is being used, and based on that experience the user will evaluate the product or products that are being presented. Using VR in order to promote different products or services is an extension of promotional elements that have been present in virtual 3D world in games, and have been introduced in virtual world with moderate to high success (van Berlo, van Reijmersdal, Smit, \& van der Laan, 2021). The authors mention that there are emotional responses related to the products that have been presented in their research and that brands attached to those products elicit a stronger response.

Taking into account these aspects we consider that we should discuss the main advantages and disadvantages that this technology has when discussing its use for promotional purposes, be it in tourism, games or any other field that a marketer would use it. The main advantages that we have identified are the following:

- Immersive worlds - The worlds created for VR are becoming more and more detailed, which allows designer and creators to have a larger freedom when compared to AR solutions. Given the possibility of recreating real life locations or creating totally new worlds, they can promote various products or services in that world either directly or indirectly, based on their approach.

- Reusable assets - when considering building a digital world, and promoting certain objects, products or services through such a world, the concept of reusing digital assets comes into play for multiple reasons. In a virtual world, depending on its size, assets can be multiplied and placed wherever target group analyses show that users are focusing their attention more. The same assets can be, in theory, moved to other similar worlds and reused, and as such the need for creating or generating new marketing materials is lower in digital worlds.

- Cost efficiency - In relation to the financial aspect that was covered with the assets, these have a mediocre cost compared to real world marketing materials and campaigns. The costs are low in comparison for the time being, not having a large audience. We expect these costs to grow in the following years as the use of this technology will become mainstream.

- Environment friendly - marketing strategies that take into account virtual worlds have an advantage of lowering the cost to the environment, due to elements being present mostly in a digital world. These digital products will not be processed or have a recycling procedure/strategy attached to them. The only impact on the environment impact that can be measured is that of the power usage by the team that designs these elements and the end users. 
These are the main advantages we have identified that can be linked with marketing strategies that want to employ the technology. There are also disadvantages when discussing the use of this technology, mostly related to end users, as follows:

- High entry costs - Depending on the quality of the worlds and their complexity, the entry cost per user can vary from tens of dollars or euros to the order of hundreds or even thousands. As we mentioned earlier, this technology can be used with either with mobile devices or computers. The first option is financially less strenuous on the user, having a requirement of a mobile devices with an age ranging between 1 to 5 years and a pair of VR glasses for that, which can be cheap or expensive depending on their quality. Still, the entry costs are lower than a full VR system, which requires a computer with VR capable graphics cards, adequate memory and processor.

- Digital skill requirements - As it was in the case of AR, the problem is more prevalent when using VR technologies, at least when full VR systems are involved, requiring hardware knowledge to configure the machines and software knowledge from the user to configure the controllers and the headset. This translates into the fact that people with low digital skills will encounter issues in use and configuration.

- Low consumer numbers - Being a niche technology, that requires additional hardware to use, the number of VR users compared to AR users are lower.

Having mentioned the advantages and disadvantages we can now present our opinion in regards to how VR can be used in marketing strategies and in promoting tourism:

- Taking into account the fact that 3D scanning has been used to preserve certain tourist attractions for further generations, or even more, digital reconstruction of such tourist attractions we consider that this is the right path to take in promoting tourism. Not all tourist attractions can be digitally reproduces at this moment, and the experience of being physically present in that location is difficult to reenact, but most of the buildings that represent some of the tourist revenue of certain cities can be digitally mapped and rebuilt. Examples can be Notre Dame de Paris, which was digitally reconstructed from previous footage, for video games that used the location for their games etc. During these virtual tourism presentations, marketers can use the occasion to promote events, locations, real life services for that location, through various methods such as screen pop-ups when looking at certain objects, planting banners in key viewing angles.

- Promoting through VR video games, which has been done in the past in titles like FIFA or GTA series. This kind of strategies should take into account the limitation of the technology right now and the lack of video games that can implement this. Although, this limitation is based on the fact that there are not many VR titles that have attracted public attention, the only mainstream notable being Half Life: Alyx, a game developed by Valve Corporation in 2020, which had a positive impact on how users perceive VR games and what they can offer. As such, In the future, the technology will allow creators and developers to use real life locations in their VR worlds, and with that element of real life locations included, the path for marketing strategies will be easier to follow through.

\section{Conclusions}

Virtual Reality and Augmented Reality are technologies that have been used throughout the years for different purposes, varying from education to medical and even promotional fields. As such, as society will interact more and more with such technologies we consider that the need to take them into consideration in marketing strategies will arise more often. The experience we are gaining 
right now through the use of technology due to lockdowns in different regions in the world will prove to be of use in the years to come, and in how we interact with technology. AR and VR technologies will become a normal element that should be present in marketing fields when it comes to presenting either products or services on various devices, be it on mobile or computers. The existing literature points towards mostly positive impact that these technologies have had on the field where they were used, and we consider that these fields should not be the only ones benefitting from this. We consider that a follow-up paper is required to analyze the impact that these technologies have had in this period, mainly focusing on tourism and marketing, since these are the fields that seem to be on the list of most affected by the pandemic situation.

\section{References}

Andreea, F., S., Remus I., H., Mihaela A., P., \& Nica, A. (2021). Virus tinged? Exploring the facets of virtual reality use in tourism as a result of the COVID-19 pandemic, Telematics and Informatics, 101575.

Cuendet, S., Bonnard, Q., Do-Lenh, S., \& Dillenbourg, P. (2013). Designing augmented reality for the classroom, Computers and Education, 557-569.

European Commission (2017, October 19). The Digital Skills Gap in Europe. Retrieved from ec.europa.eu: https://ec.europa.eu/digital-single-market/en/news/digital-skills-gap-europe

EuroStat (2021, January 29). Quarterly registrations of new businesses and declarations of bankruptcies - statistics. Retrieved from Eurostat: https://ec.europa.eu/eurostat/statisticsexplained/index.php?title=Quarterly_registrations_of_new_businesses_and_declarations_ of_bankruptcies_-_statistics\&stable=0\&redirect=no.

Joda, T., Gallucci, G., Wismeijer, D., \& Zitzmann, N. (2019). Augmented and virtual reality in dental medicine: A systematic review, Computers in Biology and Medicine, 93-100.

Kardong-Edgren, S., Farra, S., Alinier, G., \& Young, H. (2019). A Call to Unify Definitions of Virtual Reality, Clinical Simulation in Nursing, 28-34.

Milgram, P., \& Kishino, F. (1994). A Taxonomy of Mixed Reality Visual Displays. IEICE Transactions on Information Systems.

Persefoni, K., \& Tsinakos, A. (2016). A Mobile Augmented Reality Application for Primary School's History, IOSR Journal of Research \& Method in Education, 56-65.

Rauschnabel, P., Felix, R., \& Hinsch, C. (2019). Augmented reality marketing: How mobile ARapps can improve brands through inspiration, Journal of Retailing and Consumer Services, 43-53.

Rauschnabel, P., Rossmann, A., \& tom Dieck, M. (2017). An adoption framework for mobile augmented reality games: The case of Pokémon Go, Computers in Human Behavior, 276-286.

Silva, J., Southworth, M., Raptis, C., \& Silva, J. (2018). Emerging Applications of Virtual Reality in Cardiovascular Medicine, JACC: Basic to Translational Science, 420-430.

Statista (2020, January). Forecast number of mobile devices worldwide from 2020 to 2024 (in billions)*, Retrieved from statista.com: https://www.statista.com/statistics/245501/ multiple-mobile-device-ownership-worldwide/.

Sung, E. (2021). The effects of augmented reality mobile app advertising: Viral marketing via shared social experience, Journal of Business Research, 75-87.

Toubekis, G., Mayer, I., Döring-Williams, M., Maeda, K., Yamauchi, K., Taniguchi, Y., . . Jansen, M. (2017). Preservation and Management of The Unesco World Heritage Site of Bamiyan: 
Laser Scan Documentation and Virtual Reconstruction of The Destroyed Buddha Figures and The Archaeological Remains. Aachen: Universitätsbibliothek der RWTH Aachen.

van Berlo, Z., van Reijmersdal, E., Smit, E., \& van der Laan, L. (2021). Brands in virtual reality games: Affective processes within computer-mediated consumer experiences, Journal of Business Research, 458-465.

Zöllner, M., Becker, M., \& Keil, J. (2010). Snapshot Augmented Reality-Augmented Photography. The $11^{\text {th }}$ International Symposium on Virtual Reality, 53-55. 